\title{
An almost sure invariance principle for additive functionals of Markov chains
}

\author{
F. Rassoul-Agha ${ }^{\mathrm{a}}$, T. Seppäläinen ${ }^{\mathrm{b}, *}$ \\ ${ }^{a}$ Department of Mathematics, University of Utah, Salt Lake City, UT 84112, United States \\ $\mathrm{b}^{\mathrm{b}}$ Mathematics Department, University of Wisconsin-Madison, Madison, WI 53706, United States
}

Received 18 October 2006; accepted 7 September 2007

Available online 26 October 2007

\begin{abstract}
We prove an invariance principle for a vector-valued additive functional of a Markov chain for almost every starting point with respect to an ergodic equilibrium distribution. The hypothesis is a moment bound on the resolvent.
\end{abstract}

(c) 2007 Elsevier B.V. All rights reserved.

MSC: primary $60 \mathrm{~F} 17$; secondary $60 \mathrm{~J} 10$

\section{Introduction}

This note extends a result of Maxwell and Woodroofe (2000). Our notation and presentation follow Maxwell and Woodroofe (2000) as closely as possible, and some results from there will be repeated without proofs. The work presented here was motivated by applications to random walks in random environments that are reported elsewhere.

It is noteworthy that one can get the estimates needed for the invariance principles we prove by applying the theory of fractional coboundaries of Banach space contractions, developed by Derriennic and Lin (2001). Then, one only needs to assume two moments on the functional of the Markov chain ( $p=2$ below). This work, however, demonstrates how elementary probabilistic arguments give the same result if we have a slightly better moment assumption as in Maxwell and Woodroofe (2000) ( $p>2$ below).

Let $\left(X_{n}\right)_{n \geq 0}$ be a stationary ergodic Markov chain defined on a probability space $(\Omega, \mathcal{F}, P)$, with values in a general measurable space $(\mathcal{X}, \mathcal{B})$. Let $Q(x ; d y)$ be its transition probability kernel and $\pi$ the stationary marginal distribution of each $X_{n}$. Write $E$ for the expectation under $P$. $P_{x}$ denotes the probability measure obtained by conditioning on $X_{0}=x$, and $E_{x}$ is the corresponding expectation. For $p \geq 1$, we will denote by $L^{p}(\pi)$ the equivalence class of $\mathcal{B}$-measurable functions $g$ with values in $\mathbb{R}^{d}$ for some $d \geq 1$ and such that

$$
\|g\|_{p}^{p}=\int|g(x)|^{p} \pi(\mathrm{d} x)<\infty .
$$

Here, $|\cdot|$ denotes the $\ell^{2}$-norm on $\mathbb{R}^{d}$.

\footnotetext{
* Corresponding author.

E-mail addresses: firas@math.utah.edu (F. Rassoul-Agha), seppalai@math.wisc.edu (T. Seppäläinen).

URLs: http://www.math.utah.edu/ firas (F. Rassoul-Agha), http://www.math.wisc.edu/ $\sim$ seppalai (T. Seppäläinen).
} 
Now fix $d$ and an $\mathbb{R}^{d}$-valued function $g \in L^{2}(\pi)$ with $\int g \mathrm{~d} \pi=0$. Define $S_{0}(g)=0$ and

$$
S_{n+1}(g)=\sum_{k=0}^{n} g\left(X_{k}\right) \quad \text { and } \quad \widetilde{S}_{n}(g)=S_{n}(g)-E_{X_{0}}\left(S_{n}(g)\right), \quad \text { for } n \geq 0 .
$$

We are concerned with central limit type results for $S_{n}(g)$ and $\widetilde{S}_{n}(g)$. This question has been investigated from many angles and under different assumptions; see Maxwell and Woodroofe (2000) and its references. A widely used method of Kipnis and Varadhan (1986) works for reversible chains. Maxwell and Woodroofe (2000) adapted this approach to a non-reversible setting, and used growth bounds on the resolvent to obtain sufficient conditions for an invariance principle for $S_{n}(g)$ under $P$, if $p>2$.

Derriennic and Lin then used their theory of fractional coboundaries (Derriennic and Lin, 2001) to push the result to an invariance principle for $S_{n}(g)$ under $P_{x}$, for $\pi$-a.e. $x$, even when $p=2$; see Derriennic and Lin (2003). Using their method one can also show that the same almost sure invariance principle holds for $\widetilde{S}_{n}(g)$. We will show how to further the probabilistic technique of Maxwell and Woodroofe (2000) to yield both almost sure invariance principles (for $S_{n}(g)$ and $\widetilde{S}_{n}(g)$ ) when $p>2$.

Invariance principles for additive functionals of Markov chains have many applications. This note is a by-product of the authors' recent work on random walks in a random environment (Rassoul-Agha and Seppäläinen, 2005, 2006, 2007), where this invariance principle proved useful.

Let us now describe the structure of this note. In Section 2 we will present the setting of Maxwell and Woodroofe (2000) and prove an $L^{q}$ bound, with $q>2$, on a certain martingale. In Section 3 we will state and prove the main theorem of the note. The proof depends on a vector-valued version of a well-known invariance principle for martingales (Theorem 3 of Rassoul-Agha and Seppäläinen (2005)).

\section{A useful martingale}

For a function $h \in L^{1}(\pi)$ and $\pi$-a.e. $x \in \mathcal{X}$ define

$$
Q h(x)=\int h(y) Q(x ; d y) .
$$

$Q$ is a contraction on $L^{p}(\pi)$ for every $p \geq 1$. For $\varepsilon>0$ let $h_{\varepsilon}$ be the solution of

$$
(1+\varepsilon) h_{\varepsilon}-Q h_{\varepsilon}=g .
$$

In other words,

$$
h_{\varepsilon}=\sum_{k=1}^{\infty}(1+\varepsilon)^{-k} Q^{k-1} g .
$$

Note that $h_{\varepsilon} \in L^{p}(\pi)$, if $g \in L^{p}(\pi)$. On $\mathcal{X}^{2}$ define the function

$$
H_{\varepsilon}\left(x_{0}, x_{1}\right)=h_{\varepsilon}\left(x_{1}\right)-Q h_{\varepsilon}\left(x_{0}\right) .
$$

For a given realization of $\left(X_{k}\right)_{k \geq 0}$, let

$$
M_{n}(\varepsilon)=\sum_{k=0}^{n-1} H_{\varepsilon}\left(X_{k}, X_{k+1}\right) \quad \text { and } \quad R_{n}(\varepsilon)=Q h_{\varepsilon}\left(X_{0}\right)-Q h_{\varepsilon}\left(X_{n}\right)
$$

so that

$$
S_{n}(g)=M_{n}(\varepsilon)+\varepsilon S_{n}\left(h_{\varepsilon}\right)+R_{n}(\varepsilon) .
$$

Finally, let $\pi_{1}$ be the distribution of $\left(X_{0}, X_{1}\right)$ under $P$; that is

$$
\pi_{1}\left(d x_{0}, d x_{1}\right)=Q\left(x_{0} ; d x_{1}\right) \pi\left(d x_{0}\right) .
$$

Let us denote the $L^{p}$-norm on $L^{p}\left(\pi_{1}\right)$ by $\|\cdot\|_{p}$. The following theorem summarizes results of Maxwell and Woodroofe (2000). 
Theorem MW. Assume that $g \in L^{2}(\pi)$ and that there exists an $\alpha \in(0,1 / 2)$ such that

$$
\left\|\sum_{k=0}^{n-1} Q^{k} g\right\|_{2}=\sigma\left(n^{\alpha}\right) .
$$

Then:

(a) The limit $H=\lim _{\varepsilon \rightarrow 0^{+}} H_{\varepsilon}$ exists in $L^{2}\left(\pi_{1}\right)$. Moreover, if one defines

$$
M_{n}=\sum_{k=0}^{n-1} H\left(X_{k}, X_{k+1}\right),
$$

then, for $\pi$-almost every $x,\left(M_{n}\right)_{n \geq 1}$ is a $P_{x}$-square integrable martingale, relative to the filtration $\left\{\mathcal{F}_{n}=\right.$ $\left.\sigma\left(X_{0}, \ldots, X_{n}\right)\right\}_{n \geq 0}$.

(b) One has $\left\|h_{\varepsilon}\right\|_{2}=\sigma\left(\varepsilon^{-\alpha}\right)$, and if $R_{n}=S_{n}(g)-M_{n}=M_{n}(\varepsilon)-M_{n}+\varepsilon S_{n}\left(h_{\varepsilon}\right)+R_{n}(\varepsilon)$, then

$$
E\left(\left|R_{n}\right|^{2}\right)=\sigma\left(n^{2 \alpha}\right) .
$$

Proof. The existence of $H$ follows from Proposition 1 of Maxwell and Woodroofe (2000). The statement about $M_{n}$ follows from Theorem 1 therein. The bounds on $\left\|h_{\varepsilon}\right\|_{2}$ and $E\left(\left|R_{n}\right|^{2}\right)$ follow from Lemma 1 and Corollary 4 of Maxwell and Woodroofe (2000), respectively.

If, moreover, one has an $L^{p}$ assumption on $g$, then one can say more.

Theorem 1. Assume that there exists an $\alpha<1 / 2$ for which (2.1) is satisfied. Assume also that there exists a $p>2$ such that $g \in L^{p}(\pi)$. Then there exists a $q \in(2, p)$ such that $H \in L^{q}\left(\pi_{1}\right)$ and $\left(M_{n}\right)_{n \geq 1}$ is an $L^{q}$-martingale.

Proof. First choose a positive $q<(3-2 \alpha) p /(1-2 \alpha+p)$. One can check that since $2 \alpha<1$ and $p>2$, we have $q \in(2, p)$. Using Hölder's inequality, we have

$$
\left\|H_{\delta}-H_{\varepsilon}\right\|_{q}^{q} \leq\left\|H_{\delta}-H_{\varepsilon}\right\|_{p}^{a}\left\|H_{\delta}-H_{\varepsilon}\right\|_{2}^{b},
$$

where $a=p(q-2) /(p-2)<q$ and $b=q-a$. Next, observe that

$$
\left\|h_{\varepsilon}\right\|_{p} \leq \sum_{n \geq 1}(1+\varepsilon)^{-n}\|g\|_{p}=\|g\|_{p} \varepsilon^{-1} .
$$

Thus, one has

$$
\left\|H_{\delta}-H_{\varepsilon}\right\|_{q}^{q} \leq 2^{a}\|g\|_{p}^{a}\left(\varepsilon^{-1}+\delta^{-1}\right)^{a}\left\|H_{\delta}-H_{\varepsilon}\right\|_{2}^{b},
$$

and, by Lemma 2 of Maxwell and Woodroofe (2000),

$$
\left\|H_{\delta_{k}}-H_{\delta_{k-1}}\right\|_{q}^{q} \leq C 2^{k a} \cdot 2^{-k b / 2}\left(\left\|h_{\delta_{k}}\right\|_{2}^{2}+\left\|h_{\delta_{k-1}}\right\|_{2}^{2}\right)^{b / 2},
$$

where $\delta_{k}=2^{-k}$. By part (ii) of Theorem MW, we know that $\left\|h_{\delta}\right\|_{2}=\sigma\left(\delta^{-\alpha}\right)$. Therefore, one has

$$
\left\|H_{\delta_{k}}-H_{\delta_{k-1}}\right\|_{q}^{q} \leq C 2^{k(a-b / 2+\alpha b)},
$$

with maybe a different $C$ than above. Now, by the choice of $q$, one can verify that $a-b / 2+\alpha b<0$, and then repeat the proof of Proposition 1 in Maxwell and Woodroofe (2000), with $\|\cdot\|_{2}$ replaced by $\|\cdot\|_{q}$.

Remark 1. Note that Maxwell and Woodroofe (2000) uses $\|\cdot\|_{1}$ for the $L^{2}$-norm under $\pi_{1}$, while we use $\|\cdot\|_{2}$.

\section{The almost sure invariance principle}

First some notation. We write $A^{\mathrm{T}}$ for the transpose of a vector or matrix $A$. An element of $\mathbb{R}^{d}$ is regarded as a $d \times 1$ matrix, or column vector. Define

$$
\mathbb{B}_{n}(t)=n^{-1 / 2} S_{[n t]}(g) \quad \text { and } \quad \widetilde{\mathbb{B}}_{n}(t)=n^{-1 / 2} \widetilde{S}_{[n t]}(g), \quad \text { for } t \in[0,1] .
$$


Here, $[x]=\max \{k \in \mathbb{Z}: k \leq x\}$. Let $D_{\mathbb{R}^{d}}([0,1])$ denote the space of right continuous functions on $[0,1]$ taking values in $\mathbb{R}^{d}$ and having left limits. This space is endowed with the usual Skorohod topology Billingsley (1999). Let $\Delta$ denote the Prohorov metric on the space of Borel probability measures on $D_{\mathbb{R}^{d}}([0,1])$.

For a given symmetric, non-negative definite $d \times d$ matrix $\Gamma$, a Brownian motion with diffusion matrix $\Gamma$ is the $\mathbb{R}^{d}$-valued process $\{W(t): 0 \leq t \leq 1\}$ such that $W(0)=0, W$ has continuous paths, independent increments, and for $s<t$ the $d$-vector $W(t)-W(s)$ has Gaussian distribution with mean zero and covariance matrix $(t-s) \Gamma$. If the rank of $\Gamma$ is $m$, one can produce such a process by finding a $d \times m$ matrix $\Lambda$ such that $\Gamma=\Lambda \Lambda^{\mathrm{T}}$, and by defining $W(t)=\Lambda B(t)$ where $B$ is an $m$-dimensional standard Brownian motion.

Let $\Phi_{\Gamma}$ denote the distribution of Brownian motion with diffusion matrix $\Gamma$ on the space $D_{\mathbb{R}^{d}}([0,1])$. For $x \in \mathcal{X}$ let $\Psi_{n}(x)$ and $\widetilde{\Psi}_{n}(x)$, respectively, be the distributions of $\mathbb{B}_{n}$ and $\widetilde{\mathbb{B}}_{n}$ on the Borel sets of $D_{\mathbb{R}^{d}}([0,1])$ under the measure $P_{x}$; that is, conditioned on $X_{0}=x$.

Here is our main theorem.

Theorem 2. Assume there are $p>2$ and $\alpha<1 / 2$ for which $g \in L^{p}(\pi)$ and $E\left(\left|R_{n}^{2}\right|\right)=\sigma\left(n^{2 \alpha}\right)$. Then

$$
\lim _{n \rightarrow \infty} \Delta\left(\Phi_{\mathfrak{D}}, \Psi_{n}(x)\right)=0 \text { for } \pi \text {-a.e. } x \text {, }
$$

where $\mathfrak{D}=E\left(M_{1} M_{1}^{\mathrm{T}}\right)=\int H H^{\mathrm{T}} \mathrm{d} \pi_{1}$.

Remark 2. The above result improves Theorem 2 of Maxwell and Woodroofe (2000) which stated that

$$
\lim _{n \rightarrow \infty} \int \Delta\left(\Phi_{\mathfrak{D}}, \Psi_{n}(x)\right) \pi(\mathrm{d} x)=0
$$

Remark 3. Due to Theorem MW, (2.1) guarantees the bound on $E\left(\left|R_{n}\right|^{2}\right)$ in Theorem 2 .

Proof. The proof is essentially done in Maxwell and Woodroofe (2000). We explain below how to apply the Borel-Cantelli Lemma to strengthen their result to an almost sure statement.

Let $M_{n}^{*}(t)=n^{-1 / 2} M_{[n t]}$. We have

$$
\sup _{0 \leq t \leq 1}\left|\mathbb{B}_{n}(t)-M_{n}^{*}(t)\right| \leq n^{-1 / 2} \max _{k \leq n}\left|R_{k}\right| .
$$

Therefore to conclude the proof we need to show two things:

for $\pi$-almost every $x$, under the probability measure $P_{x}$ the processes

$M_{n}^{*}$ converge weakly to a Brownian motion with diffusion matrix $\mathfrak{D}$,

and

$$
n^{-1 / 2} \max _{k \leq n}\left|R_{k}\right| \underset{n \rightarrow \infty}{\longrightarrow} 0 \text { in } P_{x} \text {-probability, for } \pi \text {-a.e. } x \text {. }
$$

Statement (3.1) follows from the martingale invariance principle stated as Theorem 3 in Rassoul-Agha and Seppäläinen (2005). The limits needed as hypotheses for that theorem follow from ergodicity and the squareintegrability of $H$. We leave this check to the reader.

To prove (3.2), let $n_{j}=j^{r}$ for a large enough integer $r$. Fix $0<\gamma<1$, and let $m_{j}=\left\lceil n_{j}^{1-\gamma}\right\rceil, \ell_{j}=\left\lceil n_{j}^{\gamma}\right\rceil$. Here $\lceil x\rceil=\min \{n \in \mathbb{Z}: x \leq n\}$. Since $R_{n}=S_{n}(g)-M_{n}$, one can write

$$
\begin{aligned}
n_{j}^{-1 / 2} \max _{i \leq n_{j}}\left|R_{i}\right| \leq & n_{j}^{-1 / 2} \max _{0 \leq k \leq m_{j}}\left|R_{k \ell_{j}}\right|+n_{j}^{-1 / 2} \max _{0 \leq k<m_{j}} \max _{k \ell_{j} \leq i \leq(k+1) \ell_{j}}\left|M_{i}-M_{k \ell_{j}}\right| \\
& +n_{j}^{-1 / 2} \max _{0 \leq k<m_{j}} \max _{k \ell_{j} \leq i \leq(k+1) \ell_{j}}\left|S_{i}(g)-S_{k \ell_{j}}(g)\right| .
\end{aligned}
$$

Recalling that $E\left(\left|R_{n}\right|^{2}\right)=\sigma\left(n^{2 \alpha}\right)$ with $\alpha<1 / 2$, one can apply Corollary 3 of Maxwell and Woodroofe (2000) to get that for any $\delta>0$

$$
P\left(\max _{0 \leq k \leq m_{j}}\left|R_{k \ell_{j}}\right| \geq \delta \sqrt{n_{j}}\right)=\sigma\left(\ell_{j}^{2 \alpha} m_{j}^{\beta} / n_{j}\right)=\sigma\left(j^{-r(1-2 \gamma \alpha-(1-\gamma) \beta)}\right),
$$


for any $\beta>1$. Choosing $\beta$ close enough to 1 and $r$ large enough, the above becomes summable. The Borel-Cantelli Lemma implies then that the first term on the right-hand side of (3.3) converges to $0, P$-a.s.

The second martingale term on the right-hand side of (3.3) tends to 0 in $P_{x}$-probability for $\pi$-a.e. $x$, by the functional central limit theorem for $L^{2}$-martingales; see Theorem 3 of Rassoul-Agha and Seppäläinen (2005), for example. So it all boils down to showing that the last term in (3.3) goes to $0 P$-a.s.

Remark 4. Note that we have so far used the fact that $g \in L^{2}(\pi)$. It is only to control the third term in (3.3) that we need a higher moment.

Define, for $\delta>0$,

$$
B_{j}^{\prime}=\left\{\max _{0 \leq k<m_{j}} \max _{k \ell_{j} \leq i \leq(k+1) \ell_{j}}\left|S_{i}(g)-S_{k \ell_{j}}(g)\right| \geq \delta \sqrt{n_{j}}\right\} .
$$

Since $g \in L^{p}(\pi)$, one can write

$$
\begin{aligned}
P\left(B_{j}^{\prime}\right) & \leq P\left(\max _{i \leq n_{j}}\left|g\left(X_{i}\right)\right| \geq \delta \sqrt{n_{j}} / \ell_{j}\right) \\
& \leq n_{j} \pi\left(|g| \geq \delta \sqrt{n_{j}} / \ell_{j}\right)=\sigma\left(j^{-r(p / 2-1-\gamma p)}\right) .
\end{aligned}
$$

By choosing $\gamma$ small enough and $r$ large enough, one can make sure that $P\left(B_{j}^{\prime}\right)$ is summable. By the Borel-Cantelli Lemma, the third term in (3.3) converges to $0, P$-a.s.

Finally, note that if $n_{j-1} \leq n \leq n_{j}$, then

$$
\max _{k \leq n} \frac{\left|R_{k}\right|}{\sqrt{n}} \leq\left(\frac{j}{j-1}\right)^{r / 2} \max _{k \leq n_{j}} \frac{\left|R_{k}\right|}{\sqrt{n_{j}}},
$$

and so (3.2) follows.

Remark 5. In the above proof we only needed the martingale term in (3.3) to converge in $P_{x}$-probability. The $L^{q}$ bounds of Theorem 1 imply that it actually goes to $0 P$-a.s., making (3.2) also true $P$-a.s. All this is of course under the assumptions $p>2$ and (2.1) with $\alpha<1 / 2$. In Derriennic and Lin (2003) it is shown that the same almost sure convergence happens even when $p=2$.

We also have a similar result for $\widetilde{S}_{n}(g)$ :

Theorem 3. Assume there are $p>2$ and $\alpha<1 / 2$ for which $g \in L^{p}(\pi)$ and condition (2.1) is satisfied. Then $n^{-1 / 2} \max _{k \leq n}\left|E_{x}\left(S_{k}(g)\right)\right|$ converges to 0 as $n$ goes to infinity for $\pi$-almost every $x$. Consequently, for $\pi$-almost every $x$,

$$
\lim _{n \rightarrow \infty} n^{-1 / 2} \max _{k \leq n}\left|S_{k}(g)-\widetilde{S}_{k}(g)\right|=0 \quad P_{x} \text {-almost surely, }
$$

and, therefore,

$$
\lim _{n \rightarrow \infty} \Delta\left(\Phi_{\mathfrak{D}}, \widetilde{\Psi}_{n}(x)\right)=0 \text { for } \pi \text {-a.e. } x .
$$

The diffusion matrix $\mathfrak{D}$ is as defined in Theorem 2.

Before we start the proof, we need to re-prove a maximal inequality of Maxwell and Woodroofe (2000), this time for a Markov transition operator rather than a shift. For a probability transition kernel $Q$ and a function $g$ in its domain, define $T_{n}(g, Q)=\sum_{k=0}^{n-1} Q^{k} g$. We then have the following:

Proposition 1. Let $Q$ be a probability transition kernel with invariant measure $\pi$. Let $g \in L^{2}(\pi)$ be such that

$$
\int\left|T_{n}(g, Q)\right|^{2} \mathrm{~d} \pi \leq C(g, Q) n,
$$

for some $C(g, Q)<\infty$ and all $n \geq 1$. Then we have 


$$
\pi\left(\max _{j \leq n}\left|T_{j}(g, Q)\right|>\lambda\right) \leq \frac{2^{6 k} C(g, Q) n^{1+2^{-k}}}{\lambda^{2}},
$$

for all $n \geq 1, k \geq 0$, and $\lambda>0$.

Proof. We will proceed by induction on $k$. For $k=0$ the lemma follows from Chebyshev's and Jensen's inequalities, as well as the invariance of $\pi$ under $Q$. Let us assume that the lemma has been proved for some $k \geq 0$. We will prove it for $k+1$. To this end, choose $n \geq 1$ and $\lambda>0$. Let $m=\lceil\sqrt{n}\rceil$. Then $[n / m] \leq m$, and

$$
\begin{aligned}
\pi\left(\max _{j \leq n}\left|T_{j}(g, Q)\right|>\lambda\right) \leq & \pi\left(\max _{i \leq n / m}\left|T_{i m}(g, Q)\right|>\lambda / 2\right) \\
& +m \max _{i \leq n / m} \pi\left(\max _{j \leq m}\left|T_{j+i m}(g, Q)-T_{i m}(g, Q)\right|>\lambda / 2\right) \\
\leq & \pi\left(\max _{i \leq m}\left|T_{i}\left(T_{m}(g, Q), Q^{m}\right)\right|>\lambda / 2\right)+m \max _{i \leq m} \pi\left(\max _{j \leq m}\left|T_{j}\left(Q^{i m} g, Q\right)\right|>\lambda / 2\right) \\
\leq & \frac{4 \cdot 2^{6 k} C\left(T_{m}(g, Q), Q^{m}\right) m^{1+2^{-k}}}{\lambda^{2}}+\max _{i \leq m} \frac{4 \cdot 2^{6 k} C\left(Q^{i m} g, Q\right) m^{2+2^{-k}}}{\lambda^{2}} .
\end{aligned}
$$

But one has

$$
\int\left|T_{n}\left(T_{m}(g, Q), Q^{m}\right)\right|^{2} \mathrm{~d} \pi=\int\left|T_{m n}(g, Q)\right|^{2} \mathrm{~d} \pi \leq C(g, Q) m n
$$

and, therefore, $C\left(T_{m}(g, Q), Q^{m}\right) \leq C(g, Q) m$. Similarly,

$$
\begin{aligned}
\int\left|T_{n}\left(Q^{i m} g, Q\right)\right|^{2} \mathrm{~d} \pi & =\int\left|Q^{i m} T_{n}(g, Q)\right|^{2} \mathrm{~d} \pi \leq \int Q^{i m}\left|T_{n}(g, Q)\right|^{2} \mathrm{~d} \pi \\
& =\int\left|T_{n}(g, Q)\right|^{2} \mathrm{~d} \pi \leq C(g, Q) n .
\end{aligned}
$$

Thus, $C\left(Q^{i m} g, Q\right) \leq C(g, Q)$. Above, we have used Jensen's inequality to bring $Q$ outside the square and then the fact that $\pi$ is invariant under $Q$. Now, we have

$$
\pi\left(\max _{j \leq n}\left|T_{n}(g, Q)\right|>\lambda\right) \leq \frac{8 \cdot 2^{6 k} C(g, Q) m^{2+2^{-k}}}{\lambda^{2}} .
$$

Since $m \leq 2 \sqrt{n}$, it follows that

$$
\pi\left(\max _{j \leq n}\left|T_{n}(g, Q)\right|>\lambda\right) \leq \frac{2^{6 k+6} C(g, Q) n^{1+2^{-k-1}}}{\lambda^{2}}
$$

which is the claim of the lemma, for $k+1$.

The following is then immediate:

Corollary 1. For any $\beta>1$ there is a constant $\Gamma$, depending only on $\beta$, for which

$$
\pi\left(\max _{j \leq n}\left|T_{j}(g, Q)\right|>\lambda\right) \leq \frac{\Gamma C(g, Q) n^{\beta}}{\lambda^{2}}
$$

for all $\lambda>0$ and $n \geq 1$.

We can now prove the theorem.

Proof of Theorem 3. Observe that $E_{x}\left(S_{n}(g)\right)=T_{n}(g, Q)$. Now, recall that $n_{j}=j^{r}$, for an integer $r$ large enough. Also, for $0<\gamma<1$ we have $m_{j}=\left\lceil n_{j}^{1-\gamma}\right\rceil, \ell_{j}=\left\lceil n_{j}^{\gamma}\right\rceil$. Then,

$$
\begin{aligned}
n_{j}^{-1 / 2} \max _{i \leq n_{j}}\left|E_{x}\left(S_{i}(g)\right)\right| \leq & n_{j}^{-1 / 2} \max _{k \leq m_{j}}\left|E_{x}\left(S_{k \ell_{j}}(g)\right)\right| \\
& +n_{j}^{-1 / 2} \max _{k<m_{j} k \ell_{j} \leq i \leq(k+1) \ell_{j}}\left|E_{x}\left(S_{i}(g)\right)-E_{x}\left(S_{k \ell_{j}}(g)\right)\right| .
\end{aligned}
$$


For the first term, we can use the above corollary to write

$$
\begin{aligned}
\pi\left(n_{j}^{-1 / 2} \max _{k \leq m_{j}}\left|E_{x}\left(S_{k \ell_{j}}(g)\right)\right|>\varepsilon\right) & =\pi\left(n_{j}^{-1 / 2} \max _{k \leq m_{j}}\left|T_{k \ell_{j}}(g, Q)\right|>\varepsilon\right) \\
& =\pi\left(\max _{k \leq m_{j}}\left|T_{k}\left(T_{\ell_{j}}(g, Q), Q^{\ell_{j}}\right)\right|>\varepsilon \sqrt{n_{j}}\right) \\
& \leq \frac{\Gamma C\left(T_{\ell_{j}}(g, Q), Q^{\ell_{j}}\right) m_{j}^{\beta}}{\varepsilon^{2} n_{j}} \\
& \leq \frac{\Gamma C \ell_{j}^{2 \alpha} m_{j}^{\beta}}{\varepsilon^{2}}=\sigma\left(j^{-r(1-2 \alpha \gamma-(1-\gamma) \beta)}\right)
\end{aligned}
$$

since

$$
\int\left|T_{n}\left(T_{\ell_{j}}(g, Q), Q^{\ell_{j}}\right)\right|^{2} \mathrm{~d} \pi=\int\left|T_{n \ell_{j}}(g, Q)\right|^{2} \mathrm{~d} \pi \leq C\left(n \ell_{j}\right)^{2 \alpha} \leq C \ell_{j}^{2 \alpha} n,
$$

by (2.1). If one chooses $\beta$ small enough and $r$ large enough, then the term on line (3.5) goes to $0, \pi$-a.s., by the Borel-Cantelli Lemma. For the term on line (3.6) we have

$$
\begin{aligned}
& \pi\left(n_{j}^{-1 / 2} \max _{k<m_{j}} \max _{j \ell_{j} \leq i \leq(k+1) \ell_{j}}\left|E_{x}\left(S_{i}(g)\right)-E_{x}\left(S_{k \ell_{j}}(g)\right)\right| \geq \varepsilon\right) \\
& \quad \leq \pi\left(\max _{i \leq n_{j}}\left|Q^{i} g\right| \geq \varepsilon \sqrt{n_{j}} / \ell_{j}\right) \\
& \quad \leq n_{j} \max _{i \leq n_{j}} \pi\left(\left|Q^{i} g\right| \geq \varepsilon \sqrt{n_{j}} / \ell_{j}\right) \\
& \leq \sigma\left(j^{-r(p / 2-1-\gamma p)}\right),
\end{aligned}
$$

since

$$
\int\left|Q^{i} g\right|^{p} \mathrm{~d} \pi \leq \int Q^{i}\left(|g|^{p}\right) \mathrm{d} \pi=\int|g|^{p} \mathrm{~d} \pi<\infty .
$$

Using the Borel-Cantelli Lemma, we get that the term on line (3.6) also converges to $0, \pi$-a.s., if one chooses $\gamma$ small enough and $r$ large enough.

Therefore, we have shown that $n_{j}^{-1 / 2} \max _{i \leq n_{j}}\left|E_{x}\left(S_{i}(g)\right)\right|$ converges to $0, \pi$-a.s. The claim of the theorem follows then as in (3.4), on considering $n_{j} \leq n \leq n_{j+1}$.

\section{Acknowledgement}

T. Seppäläinen was partially supported by National Science Foundation grant DMS-0402231.

\section{References}

Billingsley, P., 1999. Convergence of Probability Measures, second edition. John Wiley \& Sons Inc., New York.

Derriennic, Y., Lin, M., 2001. Fractional Poisson equations and ergodic theorems for fractional coboundaries. Israel J. Math. 123, 93-130.

Derriennic, Y., Lin, M., 2003. The central limit theorem for Markov chains started at a point. Probab. Theory Related Fields 125 (1), 73-76.

Kipnis, C., Varadhan, S.R.S., 1986. Central limit theorem for additive functionals of reversible Markov processes and applications to simple exclusions. Comm. Math. Phys. 104 (1), 1-19.

Maxwell, M., Woodroofe, M., 2000. Central limit theorems for additive functionals of Markov chains. Ann. Probab. 28 (2), $713-724$.

Rassoul-Agha, F., Seppäläinen, T., 2005. An almost sure invariance principle for random walks in a space-time random environment. Probab. Theory Related Fields 133 (3), 299-314.

Rassoul-Agha, F., Seppäläinen, T., 2006. Ballistic random walk in a random environment with a forbidden direction. ALEA Lat. Am. J. Probab. Math. Stat. 1, 111-147 (Electronic).

Rassoul-Agha, F., Seppäläinen, T., 2007. Quenched invariance principle for multidimensional ballistic random walk in a random environment with a forbidden direction. Ann. Probab. 35 (1), 1-31. 